\title{
Prognóstico da competência percebida ATRAVÉS DA MOtivaçÃo EM PRATICANTES DE EXERCí́cIO FísICO
}

Juan Antonio Moreno Murcia' morenomu@um.es

Alvaro Sicilia Camacho²asicilia@ual.es

José María Muyor Rodríguez² josemuyor@ual.es

doi:10.3900/fpi.7.6.357.p

Murcia JAM, Camacho AS, Rodríguez JMM. Prognóstico da competência percebida através da motivação em praticantes de exercício físico. Fit Perf J. 2008 nov-dez;7(6):357-65.

\section{RESUMO}

Introdução: $\bigcirc$ objetivo principal deste trabalho foi comprovar o poder de prognóstico do clima motivacional, a orientação disposicional e a motivação autodeterminada sobre a competência percebida em praticantes de exercício físico em centros de fitness. Materiais e Métodos: Foram medidos sobre 727 praticantes de exercício físico, o clima motivacional, a orientação, a disposição, motivação autodeterminada e a competência motora percebida. Resultados: Os resultados mostraram que a percepção de um clima que implicava ao ego apresentou uma relação negativa e significativa com a percepção de um clima que implicava à tarefa, e uma relação negativa e significativa com a orientação motivacional de aproximação ao rendimento. Também foi encontrada uma associação positiva e significativa entre a percepção de um clima que implicava à mestria e as orientações motivacionais de aproximação à mestria e evitação do rendimento. Discussão: A análise de regressão percebeu que tanto a orientação de aproximação ao rendimento como à mestria e o regulamento intrínseco foram os fatores que predisseram com maior força a competência motora percebida. Por sexo, os homens apresentavam um maior clima e aproximação ao ego, maior percepção de competência, enquanto as mulheres obtiveram maiores valores no clima tarefa e motivação intrínseca. Foram discutidos os resultados em relação ao prognóstico da competência percebida em praticantes de exercício físico.

\section{PALAVRAS-CHAVE}

Motivação, Exercício, Academias de Ginástica.

\footnotetext{
1 Universidad de Murcia - Facultad de Ciencias del Deporte - Murcia - Espanha

2 Universidad de Almería - Almería - Espanha
}

Copyright(C 2008 por Colégio Brasileiro de Atividade Física, Saúde e Esporte

Fit Perf J | Rio de Janeiro | 7 | 6 | 357-365 | nov/dez 2008 


\section{Prognostic of the perceived competence through motivation In PRACTItIONers of Physical eXeRCISE}

\section{ABSTRACT}

Introduction: The main objective of this work was to prove the prognostic power of the motivational atmosphere, the dispositional orientation and the self-determined motivation about the perceived competence in physical exercises practitioners in fitness centers. Materials and Methods: We measured, on 727 physical practitioners of exercises, the motivational atmosphere, the orientation, the disposal, self-determined motivation and the perceived motor competence. Results: The results showed that the perception of an atmosphere that implied to the ego presented a negative and significant relation with the perception of an atmosphere which implied the task, and a negative and significant relation with the motivational orientation of approximation to efficiency. It was also found a positive and significant association between the perception of an atmosphere that implied the mastery and the motivational orientation of approximation to the mastery and avoidance to efficiency. Discussion: The analysis of the regression perceived that both the orientation to the approximation to efficiency and the mastery and the intrinsic regulation were the factors that better predicted the perceived motor competence. Concerning the sex, men presented a greater atmosphere and approximation to the ego, a better competence perception, while women got greater values in the atmosphere task and intrinsic motivation. The results regarding the prognostic of the perceived competence in practitioners of physical exercise were discussed.

\section{KEYWORDS}

Motivation, Exercise, Fitness Centers.

\section{Predicción de la competencia percibida según la motivación en Practicantes de ejercicio físico \\ RESUMEN}

Introducción: El objetivo principal de este trabajo ha sido comprobar el poder de predicción del clima motivacional, la orientación disposicional y la motivación autodeterminada sobre la competencia percibida en practicantes de ejercicio físico en centros de fitness. Materiales y Métodos: Se midieron sobre 727 practicantes de ejercicio físico el clima motivacional, la orientación, la disposición, motivación autodeterminada y la competencia motriz percibida. Resultados: Los resultados mostraron que la percepción de un clima que implicaba al ego presentó una relación negativa y significativa con la percepción de un clima que implicaba a la tarea, y una relación negativa y significativa con la orientación motivacional de aproximación al rendimiento. También se encontró una asociación positiva y significativa entre la percepción de un clima que implicaba a la maestría y las orientaciones motivacionales de aproximación a la maestría y evitación al rendimiento. Discusión: El análisis de regresión halló que tanto la orientación de aproximación al rendimiento como a la maestría y la regulación intrínseca fueron los factores que predijeron con mayor fuerza la competencia motriz percibida. Por sexo, los hombres presentaban un mayor clima y aproximación al ego, mayor percepción de competencia, mientras que las mujeres obtuvieron mayores valores en el clima tarea y motivación intrínseca. Se discutieron los resultados en relación a la predicción de la competencia percibida en practicantes de ejercicio físico.

\section{PALABRAS CLAVE}

Motivación, Ejercicio, Centros de Acondicionamiento.

\section{INTRODUÇÃO}

Atualmente, um dos fenômenos sociais que caracterizam as sociedades avançadas é o aumento da prática de exercício físico e sua generalização nos diferentes segmentos populacionais ${ }^{1}$. Além disso, a realização de exercício físico praticado de forma regular com uma intensidade, duração e descanso adequados, produz importantes benefícios, tanto em nível físico como psicológico ${ }^{2,3}$. Com isto, o autoconceito físico é considerado como um bom indicador do estado mental e social do praticante ${ }^{4,5,6}$. Apesar disso, a investigação indicou que a motivação da pessoa pode dirigir e regular a forma com que ele se percebe ${ }^{\mathbf{7}}$. Nesta linha, é interessante entender os processos motivacionais que guiam os usuários de centros esportivos e indagar sobre os possíveis efeitos que a motivação tem na autopercepção física. Assim, a Teoria da Autodeterminação ${ }^{\mathbf{8} 9}$ e a Teoria de Metas de Logro $\mathbf{1 0 , 1 1 , 1 2 , 1 3 , 1 4 , 1 5}$ foram utilizadas neste estudo para comprovar a relação que apresentam com a competência esportiva.

Para explicar a motivação físico-esportiva, os pesquisadores se basearam prioritariamente em duas grandes teorias contemporâneas, a Teoria das Metas de Logro ${ }^{15}$ e a Teoria da Autodeterminação $\mathbf{8 , 9}^{\mathbf{1 6}, \mathbf{1 7}}$. A Teoria da Autodeterminação ${ }^{\mathbf{8}, 16}$ se baseia na idéia de que o comportamento humano é motivado por três necessidades psicológicas primárias e universais: autonomia, competência e relação com os demais. Em resumo, se pode dizer 
que a autodeterminação se refere à experiência de ser livre para iniciar comportamentos ${ }^{\mathbf{8}}$. Dentro da motivação existem diferentes níveis de autodeterminação, já que esta se considera um contínuo. De maior a menor autodeterminação, apareceriam à motivação ou regulamento intrínseco, a motivação identificada, a motivação introduzida, a motivação extrínseca e a desmotivação 9,17.

A motivação intrínseca se produz quando o indivíduo realiza a tarefa pelo próprio desfrute e o prazer que lhe reporta durante a mesma, isto é, onde a atividade é o fim em si mesma ${ }^{8}$. No regulamento o sujeito se identifica com a importância que tem a atividade para si mesmo, mesmo que a prática seja um instrumento para conseguir algo ${ }^{\mathbf{1 7}}$. O regulamento introduzido faz referência a uma motivação para a prática esportiva, para evitar sentimentos de culpabilidade", isto é, se "tem" ou se "deve" fazer algo ${ }^{\mathbf{1 8}}$. Dentro do contínuo de autodeterminação, depois do regulamento introduzido estaria o regulamento extrínseco, que é o que tradicionalmente se conheceu como motivação externa. Nela, o indivíduo atua por um incentivo externo ${ }^{\mathbf{1 7}}$, como, por exemplo, a consecução do reconhecimento dos demais ou uma aprovação social. Finalmente, a desmotivação costuma se situar no extremo do contínuo porque significa a ausência absoluta de qualquer tipo de motivação. Caracteriza-se porque o indivíduo não tem nenhuma intenção de realizar a tarefa ou, em nosso caso, a prática esportiva. Normalmente, vai acompanhada de sentimentos de frustração e uma sensação que a atividade supõe já ser uma perda de tempo,16.

Com base nisso, a Teoria de Metas de Logro foi a outra grande teoria que os pesquisadores utilizaram para o estudo da motivação no campo esportivo. Sob esta teoria, o indivíduo é percebido como um organismo intencional, dirigido por uns objetivos para uma meta que opera de maneira racional ${ }^{14}$. Neste sentido, as metas do indivíduo se baseiam no esforço que têm que fazer para demonstrar competência e habilidade em contextos de logro ${ }^{\mathbf{1 3}, \mathbf{1 4}}$. Entende-se por contextos de logro aqueles contextos nos quais participa o sujeito e dos que pode receber influências para a orientação de suas metas. Assim, as pessoas diferem nas suas definições de sucesso ou fracasso quando se encontram em ambientes de logro ${ }^{19}$, e disso depende, em grande medida, sua percepção de habilidade ${ }^{15}$. Em função do conceito de habilidade adquirido pelos indivíduos, estes apresentarão uma maior implicação bem à mestria (ou também denominada tarefa) ou ao rendimento (referida também como ego). Esta implicação dependerá fundamentalmente de dois fatores: um disposicional e outro ambiental.

$\bigcirc$ fator disposicional se refere às características pessoais do indivíduo que lhe faz definir o sucesso de acordo com diferentes critérios, os quais refletiriam a diferente orientação ou perspectiva motivacional que possui. Tradicionalmente, esta teoria tinha definido duas claras perspectivas pelas quais o sujeito define seu sucesso. Por um lado, existem sujeitos que consideram haver tido sucesso quando demonstraram uma melhora pessoal em conseqüência de seu esforço. Diz-se que os sujeitos que apresentam este tipo de orientação possuem uma orientação dirigida para a tarefa ou mestria, dado que guiam a definição de seu sucesso por um claro critério referencial consigo mesmo. Por outro lado, existem sujeitos que definem seu sucesso com base numa comparação constante com outros. Neste último caso, se diz que os sujeitos apresentam uma orientação para o ego ou o rendimento, dado que estes indivíduos só costumam mostrar sucesso quando demonstram que o fazem igual ou melhor do que os demais.

Recentemente, autores como Elliot ${ }^{20}$ e Elliot \& McGregor ${ }^{21}$ indicaram que é interessante também ter em conta o grau ou direção para o qual os sujeitos se orientam. Neste sentido, os sujeitos nem sempre se centram em mostrar sua competência (bem se orientando para a tarefa ou o ego), senão que também podem estar interessados em evitar sua incompetência. Portanto, vale analisar não só quando os usuários se orientam para um ou outro tipo de motivação, como também se estes evitam se orientar a alguma delas. Neste estudo se optou por considerar a proposta que realizam Elliot ${ }^{20}$ e Elliot \& McGregor ${ }^{\mathbf{2 1}}$. Assim, dentro da Teoria de Metas de Logro, e tendo em conta o grau da orientação, existiriam quatro orientações possíveis: aproximação à tarefa ou mestria, evitação à tarefa, aproximação ao ego ou rendimento e evitação ao ego. Diz-se que, quando o sujeito tem uma orientação ao ego ou rendimento, sua concepção de habilidade se corresponde com uma entidade fixa e inata, onde julga sua capacidade em relação e comparação com os demais. $\bigcirc$ sucesso ou o fracasso depende da valoração subjetiva que resulte de comparar sua própria habilidade com os outros ${ }^{\mathbf{1 4}}$. Se a orientação do indivíduo é para a tarefa ou mestria, a concepção que o sujeito tem de sua habilidade costuma ser modificável, melhorável e treinado. Por isso, existe uma preocupação pela aprendizagem, onde as percepções de habilidade são auto-referenciais e dependem do progresso pessoal|14.

Por outro lado, o fator ambiental, representado pelo clima motivacional, pode modificar o estado particular de envolvimento do sujeito ${ }^{15}$. Assim, um ambiente ou clima motivacional que se orienta para a aprendizagem ao invés do rendimento, costuma se relacionar positivamente com as metas orientadas à tarefa, satisfação, diversão, interesse e motivação intrínseca ${ }^{\mathbf{2 2} 23}$. Pelo contrário, um clima motivacional que se orienta para o rendimento costuma estar relacionado positivamente com a orientação ao ego, com uma negativa efetividade e com sentimentos 
de pressão ${ }^{\mathbf{2 2 , 2 3}}$. $\bigcirc$ autoconceito e a auto-estima são variáveis que estão claramente unidas com a motivação ${ }^{8}$. De fato, a percepção de incompetência e baixa percepção física poderiam levar a uma falta de motivação?. Portanto, a percepção que um tem de si mesmo é outro fator determinante das situações de logro. A investigação sugeriu que as pessoas que se percebem com alto nível de competência num domínio dado (seja cognitivo, físico ou social), estão mais motivadas intrinsecamente para continuar uma atividade, e é mais provável que estejam dispostas a realizar um maior esforço durante a realização da mesma $\mathbf{2 4 , 2 5 , 2 6}$.

Particularmente, a pesquisa dentro do campo da atividade física mostra evidências que apóiam a existência de uma relação entre a competência percebida e os processos motivacionais ${ }^{\mathbf{2 7}}$. As pessoas com uma percepção alta de competência teriam mais probabilidade em eleger tarefas desafiantes, de se divertir durante o processo de aprendizagem, maior auto-estima, utilizariam critérios internos para julgar o sucesso, desenvolveriam mais esforço para a realização da atividade e seriam mais persistentes quando encontrassem uma dificuldade a superar ${ }^{\mathbf{8 , 2 4 , 2 8}}$. A estrutura teórica que rodeia, tanto a Teoria de Metas de Logro quanto a Teoria da Autodeterminação, indicou que a disposição motivacional do sujeito está fortemente relacionada com a forma em que este constrói seu nível de competência em situações de logro ${ }^{8,14,15}$. Uma pessoa intrinsecamente motivada, ou com uma orientação à tarefa, tende a se centrar na atividade que realiza, faz mais por resolver os problemas que se the apresenta, e tenta melhorar e fazê-lo melhor do que da última vez. Pelo contrário, uma pessoa externamente motivada, ou com uma motivação orientada para o ego, tende a se centrar em aspectos mais afastados da tarefa, tais como mostrar maior habilidade frente aos demais ou evitar fazê-lo pior do que outros. Portanto, as pessoas motivadas intrinsecamente e com uma orientação centrada na tarefa é provável que construam seu autoconceito sobre diferentes critérios que as pessoas com uma motivação externa, ou uma disposição orientada mais ao ego e ao rendimento.

Em função das teorias e investigações revisadas sobre a motivação para a prática de exercício físico, o principal objetivo do estudo foi indagar o efeito de prognóstico da Teoria de Metas de Logro e a Motivação Autodeterminada sobre a competência percebida. Um objetivo secundário foi analisar as diferenças por sexo nas variáveis consideradas. Partindo da revisão dos diferentes trabalhos de pesquisa, este estudo espera encontrar que os usuários que apresentam uma maior orientação motivacional para a tarefa, maior motivação intrínseca e uma percepção de clima para a tarefa, terão uma percepção mais alta de sua competência motora.

\section{MATERIAIS E MÉTODOS}

\section{Participantes}

A amostra foi composta por 727 sujeitos (402 homens e 325 mulheres), de idades compreendidas entre 14 anos e 78 anos $(m=32,57 ; d p=11,40)$, participantes de instalações desportivas públicas de uma cidade espanhola.

\section{Instrumentos}

Questionário de Medida das Estratégias Motivacionais nas Classes de Educação Física (CMEMEF) - Utilizou-se este questionário de Cervelló et al. ${ }^{29}$ adaptado a praticantes de exercício físico. Foi composto por um total de 12 itens para o clima tarefa (por exemplo, "animam-nos a que nos ajudemos entre colegas durante as tarefas") e 12 itens para o clima ego (por exemplo, "só se avalia o resultado final, sem lhe importar o progresso referido a como o fazia antes"). Encabeçava-lhe o enunciado "No centro esportivo..." e utilizava uma escala tipo Likert de 1 (totalmente em desacordo) a 7 (totalmente de acordo). A análise de confiabilidade apresentou um valor de alfa de Cronbach de 0,77 para o clima-tarefa e 0,78 para o clima-ego.

Escala de Metas de Logro 2 X 2 - Para avaliar a disposição motivacional dos usuários, se utilizou a versão espanhola da adaptação à educação física ${ }^{30}$ do Questionário de Metas de Logro 2 X $2{ }^{21}$. Este era composto por 12 itens divididos em quatro fatores de três itens cada um: meta aproximação-mestria (exemplo, "quero aprender ou melhorar o máximo possível"); meta evitação-mestria (exemplo, "às vezes tenho medo de não poder entender ou melhorar tanto como me agradaria"); meta aproximação-rendimento (exemplo, "é importante para mim que eu faça melhor do que os outros"); e meta evitação-rendimento (exemplo, "minha meta é evitar fazêlo mal"). A análise de Cronbach apresentou um valor de 0,61, 0,74 0,73 e 0,68, para cada um destes quatro fatores, respectivamente.

Questionário de Regulação da Conduta no Exercício Físico-2 (BREQ-2) - Empregou-se a versão traduzida do espanhol por Moreno et al. ${ }^{\mathbf{3 1}}$, do original Behavioral Regulation in Exercise Questionnaire-2 32 . Foi composto por 19 itens ao todo, dividido em cinco fatores (de mais a menos autodeterminada): regulação intrínseca, composta por quatro itens (exemplo, "eu faço exercício físico porque acredito que o exercício é divertido"); regulação identificada, composta por quatro itens (exemplo, "eu faço exercício físico porque valorizo os benefícios que ele tem"); regulação introduzida, dividida em três itens (exemplo, "eu faço exercício físico porque me sinto culpado quando não o pratico"); regulação externa, formada por quatro itens (exemplo, "eu faço exercício físico porque os demais me dizem que devo fazê-lo"); e a desmotiva- 
ção, composta por quatro itens (exemplo, "não vejo por que tenho que o fazer"). Dita escala é tipo Likert que vai desde 0 (nada verdadeiro) a 4 (totalmente verdadeiro). A análise de confiabilidade apresentou um valor de alfa de Cronbach de 0,76 para a regulação intrínseca, 0,59 para a regulação identificada, 0,61 para a regulação introduzida, 0,76 para a regulação externa e, 0,60 para a desmotivação.

Competência esportiva - Para medir a competência percebida foi utilizado o fator competência esportiva da versão espanhola ${ }^{\mathbf{3 3}}$ do Physical Self-Perception Profile ${ }^{\mathbf{3 4}}$. Dito fator se compunha de seis itens (exemplo, "Sou muito bom em quase todos os esportes"), que se respondem mediante uma escala tipo Likert que vai de 1, valor correspondente a totalmente em desacordo, a 4, que indicava que o aluno estava totalmente de acordo com o que se Ihe propunha. A confiabilidade foi de $\alpha=0,85$.

Vários fatores mostraram uma confiabilidade inferior ao recomendado $0,70{ }^{35}$. Contudo, dado o pequeno número de itens que compõem os fatores, a validade interna observada pode ser marginalmente aceita ${ }^{36,37}$

\section{Procedimentos}

A permissão para desenvolver esta pesquisa foi recebida por parte dos centros esportivos. Os usuários foram informados dos objetivos do estudo e de seus direitos como participantes. Todos participaram voluntariamente no desenvolvimento da pesquisa. Os instrumentos necessários para medir as variáveis do estudo foram administrados na entrada principal dos centros, na presença de dois pesquisadores formados previamente em metodologia de investigação por enquetes. Os instrumentos se deram, em todos os casos, na mesma ordem. Além disso, para cada caso, se realizava uma breve explicação dos objetivos do estudo, se informava como responder o instrumento e, em caso necessário, se solucionavam as possíveis dúvidas surgidas durante o processo. Também se fez questão do anonimato das respostas, que se respondesse com sinceridade e que se usasse o tempo necessário para responder corretamente todas as perguntas. $\bigcirc$ tempo médio requerido para preencher o questionário completo foi de $20 \mathrm{~min}$, variando segundo a idade dos usuários das instalações esportivas. Não foram encontrados problemas relevantes à hora de responder ou entender as questões propostas.

\section{Análise de dados}

Realizaram-se estatísticas descritivas de todas as variáveis e suas respectivas análises de correlação. Posteriormente, realizaram-se análise de regressão para comprovar o poder de prognóstico do clima motivacional, da orientação motivacional e a motivação autodeterminada da competência motora percebida. Para comprovar as possíveis diferenças de todas as variáveis, segundo o sexo do praticante, foi realizada uma análise de variância (ANOVA).

\section{RESULTADOS}

\section{Estatísticas descritivas e análises de correlação}

Como se pode ver na Tabela 1, os usuários pontuaram mais alto a percepção de um clima motivacional implicado à tarefa do que um clima ego. Considerando as quatro possíveis disposições motivacionais que contemplam a Teoria de Metas de Logro, os usuários obtiveram uma maior pontuação na aproximação à mestria, seguido da evitação ao rendimento, evitação à mestria e a aproximação ao rendimento. Com respeito à motivação autodeterminada, apresentaram uma alta motivação intrínseca, seguida da motivação identificada, da regulação introduzida, da regulação externa e com uma menor pontuação à desmotivação.

Quanto às correlações (Tabela 1), podemos ver que o clima ao ego apresentou uma correlação negativa e

Tabela 1 - Análise descritiva, consistência interna e correlação de todas as variáveis

\begin{tabular}{|c|c|c|c|c|c|c|c|c|c|c|c|c|c|c|c|}
\hline & $M$ & DT & Alfa & 1 & 2 & 3 & 4 & 5 & 6 & 7 & 8 & 9 & 10 & 11 & 12 \\
\hline 1. Clima ego & 2,59 & 0,87 & 0,78 & - & $-0,26^{* *}$ & $0,27^{* *}$ & $-0,14^{* *}$ & $0,18^{* *}$ & $0,13^{* *}$ & $-0,15^{* *}$ & $-0,30$ & $0,15^{* *}$ & $0,20^{* *}$ & $0,22 * *$ & $-0,09^{*}$ \\
\hline 2. Clima tarefa & 4,81 & 0,85 & 0,77 & - & - & 0,02 & $0,37^{* *}$ & $0,08^{*}$ & $0,13^{* *}$ & $0,27^{* *}$ & $0,24^{* *}$ & 0,02 & $-0,02$ & $-0,06$ & $0,24^{* *}$ \\
\hline 3. Aproximação rendimento & 3,28 & 1,60 & 0,73 & - & - & - & $0,24^{* *}$ & $0,42^{* *}$ & $0,41^{* *}$ & 0,01 & 0,07 & $0,26^{* *}$ & $0,12^{* *}$ & $0,18^{* *}$ & $0,15^{* *}$ \\
\hline 4. Aproximação mestria & 5,60 & 1,09 & 0,61 & - & - & - & - & $0,18^{* *}$ & $0,27^{* *}$ & $0,30^{* *}$ & $0,31^{* *}$ & $0,12^{* *}$ & $-0,10^{* *}$ & $-0,80^{*}$ & $0,31^{* *}$ \\
\hline 5. Evitação rendimento & 3,69 & 1,59 & 0,69 & - & - & - & - & - & $0,56^{* *}$ & 0,01 & 0,01 & $0,21^{* *}$ & $0,15^{* *}$ & $0,19^{* *}$ & 0,04 \\
\hline 6. Evitação mestria & 3,68 & 1,56 & 0,75 & - & - & - & - & - & - & 0,03 & 0,05 & $0,26^{* *}$ & $0,18^{* *}$ & $0,12^{* *}$ & $-0,03$ \\
\hline 7. Regulação intrínseca & 3,15 & 0,75 & 0,76 & - & - & - & - & - & - & - & $0,49^{* *}$ & 0,01 & $-0,21^{* *}$ & $-0,18^{* *}$ & $0,40^{* *}$ \\
\hline 8. Regulação identificada & 2,85 & 0,57 & 0,60 & - & - & - & - & - & - & - & - & $0,24^{* *}$ & $-0,10^{* *}$ & $-0,14^{* *}$ & $0,31^{* *}$ \\
\hline 9. Regulação introduzida & 1,03 & 0,94 & 0,61 & - & - & - & - & - & - & - & - & - & $0,37^{* *}$ & $0,26^{* *}$ & 0,00 \\
\hline 10. Regulação externa & 0,41 & 0,67 & 0,76 & - & - & - & - & - & - & - & - & - & - & $0,52^{* *}$ & $-0,15^{* *}$ \\
\hline 11. Desmotivação & 0,36 & 0,60 & 0,60 & - & - & - & - & - & - & - & - & - & - & - & $-0,01^{* *}$ \\
\hline 12. Competência percebida & 3,57 & 0,73 & 0,86 & - & - & - & - & - & - & - & - & - & - & - & - \\
\hline
\end{tabular}

${ }^{*} p<0,05 ;{ }^{* *} p<0,001$ 
significativa com o clima tarefa, a aproximação à mestria, a regulação intrínseca e a competência percebida. Por outro lado, o clima ego manteve uma relação positiva e significativa com a aproximação ao rendimento, com a evitação ao rendimento, a evitação à mestria, regulação introduzida, regulação externa e desmotivação. $\bigcirc$ clima tarefa, no entanto, apresentou uma relação positiva e significativa com a aproximação à mestria, a evitação ao rendimento, a evitação à mestria, a motivação intrínseca, a regulação identificada e a competência percebida.

\section{Análise de regressão}

Realizou-se uma análise de regressão em três etapas para examinar como o clima percebido, a disposição motivacional e a motivação autodeterminada afetam a competência motora percebida (Tabela 2). A competência percebida foi predita negativamente pela evitação à mestria, a regulação extrínseca e a desmotivação. No entanto, o clima tarefa, a aproximação ao rendimento, a aproximação à mestria, a regulação intrínseca e a regulação identificada predisseram positiva e significativamente a competência percebida, explicando $26 \%$ da variância total.

\section{Análise de variância segundo o sexo}

Finalmente, para examinar as diferenças por sexo em cada uma das variáveis consideradas neste estudo, se realizou uma análise de variância (Tabela 3). Obtiveramse diferenças significativas no clima ego ( $F=34,09$; $p<0,001)$, clima tarefa $(F=10,89 ; p<0,001)$, aproximação ao rendimento $(F=34,05 ; p<0,001)$, regulação intrínseca $(F=10,12 ; p<0,001)$, desmotivação $(F=8,38$; $p<0,001)$, e competência percebida $(F=5,43 ; p<0,005)$. Assim, os homens mostraram maiores valores em orientação ao ego, aproximação ao rendimento, desmotivação e competência percebida, do que as mulheres. Pelo contrário, as mulheres pontuaram mais alto do que os homens na percepção do clima tarefa e regulação intrínseca.

\section{DISCUSSÃO}

O principal objetivo do estudo foi comprovar o efeito de prognóstico da Teoria de Metas de Logro e a Motivação Autodeterminada sobre a competência percebida, além de analisar as diferenças por sexo nas variáveis consideradas.

Nossos resultados mostraram uma relação negativa e significativa entre a percepção de clima ego e clima tarefa, tal e como outros estudos já tinham indicado $\mathbf{3 8 , 3 9 , 4 0 , 4 1}$. A percepção do clima ego mostrou, além disso, uma relação positiva e significativa com a aproximação ao rendimento, em comparação com os resultados encontrados por White \& Duda ${ }^{\mathbf{4}}$, que acharam que os sujeitos orientados ao ego mostravam uma participação esportiva mais associada à competição e ao reconhecimento social. Por outro lado, o clima tarefa neste estudo se relacionou positiva e significativamente com a aproximação à mestria e à evitação ao rendimento, coincidindo com estudos como os de White \& Duda ${ }^{41}$ e Duda et al. ${ }^{\mathbf{4 2}}$.

Este estudo mostrou também que diferentes orientações motivacionais foram relacionadas com a competência motora percebida. Em verdade, os resultados revelam que tanto a orientação de aproximação ao ego ou rendimento, como a orientação de aproximação à tarefa ou aprendizagem, foram associadas positivamente com a competência motora percebida. Contudo, ambas as orientações motivacionais predisseram positivamente a competência motora percebida. Apesar de que outros estudos mostrem que uma alta orientação à tarefa está associada com padrões adaptativos ${ }^{\mathbf{1 4 , 1 5 , 4 3}}$, a relação encontrada neste estudo entre diferentes orientações motivacionais e a competência motora sugere que os significados que os usuários atribuem ao sucesso poderiam ser um fator determinante da percepção que eles têm de si mesmos. De fato, como puseram de manifesto investigações prévias ${ }^{33,44,45,46}$, a associação mais forte entre as orientações de meta e o autoconceito físico costuma se encontrar em sua dimensão da sub-escala competência esportiva.

Tabela 2 - Análise de regressão linear da competência percebida segundo a teoria de metas e a motivação autodeterminada

\begin{tabular}{|c|c|c|c|c|}
\hline Variáveis & $\mathrm{B}$ & SEB & $\beta$ & $\mathrm{Adj}, \mathrm{R}^{2}$ \\
\hline Passo 1 & 2,65 & 0,19 & & $0,05^{* *}$ \\
\hline Clima ego & $-0,02$ & 0,03 & $-0,03$ & \\
\hline Clima tarefa & 0,20 & 0,03 & $0,23^{* *}$ & \\
\hline Passo 2 & 2,06 & 0,20 & & $0,15^{* *}$ \\
\hline Clima ego & $-0,03$ & 0,03 & $-0,04$ & \\
\hline Clima tarefa & 0,13 & 0,03 & $0,15^{* *}$ & \\
\hline Aproximaçãa rendimento & 0,07 & 0,01 & $0,17^{* *}$ & \\
\hline Aproximação mestria & 0,17 & 0,02 & $0,26^{* *}$ & \\
\hline Evitação rendimento & 0,01 & 0,02 & 0,03 & \\
\hline Evitação mestria & $-0,09$ & 0,02 & $-0,20^{* *}$ & \\
\hline Passo 3 & 1,37 & 0,20 & & $0,26^{* *}$ \\
\hline Clima ego & $-0,02$ & 0,03 & $-0,03$ & \\
\hline Clima tarefa & 0,08 & 0,03 & $0,09^{*}$ & \\
\hline Aproximaçãa rendimento & 0,07 & 0,01 & $0,17^{* *}$ & \\
\hline Aproximação mestria & 0,11 & 0,02 & $0,16^{* *}$ & \\
\hline Evitação rendimento & 0,01 & 0,01 & 0,03 & \\
\hline Evitação mestria & $-0,08$ & 0,01 & $-0,17^{* *}$ & \\
\hline Regulação intrínseca & 0,26 & 0,03 & $0,27^{* *}$ & \\
\hline Regulação identificada & 0,14 & 0,05 & $0,11^{*}$ & \\
\hline Regulação introduzida & $-0,03$ & 0,02 & $-0,04$ & \\
\hline Regulaçãa externa & $-0,11$ & $-0,04$ & $-0,10^{*}$ & \\
\hline Desmotivação & 0,15 & 0,04 & $-0,12^{*}$ & \\
\hline
\end{tabular}

${ }^{*} p<0,05 ;{ }^{* *} p<0,001$ 
Com respeito à motivação autodeterminada, encontramos que a regulação intrínseca e a regulação identificada, os dois tipos de regulação mais autodeterminadas, são as que mantêm uma associação positiva mais alta com a orientação de aproximação à mestria e à percepção de um clima tarefa. Assim mesmo, ambos os tipos de regulação são as que mantêm uma associação positiva mais forte com a competência motora percebida e são, também, os tipos de regulação que, segundo nossos resultados, iriam predizer com maior força a competência motora percebida. Pelo contrário, a regulação extrínseca predisse negativamente a competência percebida, coincidindo assim com o estudo realizado por Georgiadis et al. ${ }^{\mathbf{4 7}}$

Os resultados encontrados na análise de correlação e na análise de regressão parecem estar ligados com as hipóteses defendidas desde cada uma das teorias motivacionais. De fato, a disposição motivacional da pessoa está conectada com a forma em que se percebe a si mesma e constrói seu nível de competência em situações de logro, tais como podem ser os centros esportivos ${ }^{\mathbf{8}, 14,15}$ Um usuário do centro esportivo, que tenha uma motivação intrínseca ou uma orientação à tarefa, é mais provável que se perceba a si mesmo como fisicamente competente na medida em que tem a se centrar na tarefa, tenta resolver as dificuldades encontradas e melhorar, em lugar de se molestar por conseguir metas externas ou demonstrar mais habilidade do que os demais. Pelo contrário, um usuário que se mova mais por um motivo externo, ou tenha uma orientação motivacional ao ego, é mais provável que tenha uma alta percepção de competência quando consegue a meta ou demonstra mais habilidade diante dos colegas. Portanto, dado que neste caso o sucesso está baseado em conseguir uma meta externa ou numa comparação normativa, o envolvimento pessoal na atividade não será suficiente para se ver mais competente na habilidade. Assim, um sujeito pode perceber sua competência desde referências internas ou externas, mas lhe dará mais importância a umas ou outras dependendo do tipo de orientação motivacional que apresente.

Por outro lado, se observamos os resultados que relacionam os climas motivacionais e a competência percebida, pode-se afirmar que os usuários dos centros esportivos que participaram neste estudo tiveram mais probabilidade de perceber maiores níveis de competência quando perceberam que no centro se atendia mais a sua aprendizagem em lugar do seu rendimento. Este resultado está ligado com a Teoria de Metas de Logro, dado que as pessoas seriam mais propensas a responder com uma conduta adaptativa quando o significado do logro se baseia na melhora pessoal e o esforço, mais do que no rendimento e a habilidade que se mostra ${ }^{10,11,14,15}$. fato de que a associação entre a percepção de um clima implicante ao ego e a competência motora percebida seja baixa, poderia ser explicado pela possibilidade de que um ambiente centrado no rendimento gere uma motivação naqueles usuários que se percebam mais competentes do que seus colegas ${ }^{\mathbf{1 5 , 4 3}}$. Como Reinboth \& Duda ${ }^{\mathbf{4 8}}$ mostraram, uma competência alta poderia amortecer os efeitos negativos que um ambiente centrado no rendimento teria para o estado mental do praticante. Apesar disso, estes resultados se mostram contraditórios com outras investigações que seguem mostrando a percepção de um clima implicado ao ego como um preditor negativo do autoconceito ${ }^{49}$ e como um preditor positivo do esgotamento físico e mental ${ }^{\mathbf{4 8}}$. Portanto, mais investigações seriam necessárias para identificar a forma em que um ambiente centrado no rendimento afetaria o modo em que os usuários de centros esportivos se percebem, dentro do domínio da atividade física.

Nosso segundo objetivo neste estudo foi examinar as diferenças por sexo nas variáveis estudadas. Os resultados indicaram que os homens pontuaram mais alto em orientação ao ego, aproximação ao rendimento, desmotivação e competência percebida, do que as mulheres. As mulhe-

Tabela 3 - ANOVA por gênero, segundo a teoria de metas e a motivação autodeterminada

\begin{tabular}{|c|c|c|c|c|c|c|}
\hline & \multicolumn{2}{|c|}{ homens $(v=402)$} & \multicolumn{2}{|c|}{ mulheres $(v=325)$} & \multirow[b]{2}{*}{$\mathrm{F}$} & \multirow[b]{2}{*}{$\mathrm{p}$} \\
\hline & $M$ & DT & $M$ & DT & & \\
\hline Clima ego & 2,75 & 0,04 & 2,37 & 0,04 & 34,09 & 0,000 \\
\hline Clima tarefa & 4,71 & 0,04 & 4,93 & 0,05 & 10,89 & 0,001 \\
\hline Aproximação rendimento & 3,62 & 0,07 & 2,87 & 0,09 & 34,05 & 0,000 \\
\hline Aproximação mestria & 5,67 & 0,05 & 5,52 & 0,06 & 1,32 & 0,250 \\
\hline Evitação rendimento & 3,64 & 0,08 & 3,76 & 0,09 & 0,99 & 0,319 \\
\hline Evitação mestria & 3,66 & 0,07 & 3,70 & 0,09 & 0,12 & 0,730 \\
\hline Regulação intrínseca & 3,10 & 0,04 & 3,21 & 0,04 & 10,12 & 0,002 \\
\hline Regulação identificada & 2,85 & 0,03 & 2,86 & 0,03 & 1,29 & 0,255 \\
\hline Regulação introduzida & 1,10 & 0,05 & 0,95 & 0,05 & 1,86 & 0,172 \\
\hline Regulação externa & 0,41 & 0,03 & 0,41 & 0,04 & 0,47 & 0,490 \\
\hline Desmotivação & 0,43 & 0,03 & 0,29 & 0,03 & 8,38 & 0,004 \\
\hline Competência percebida & 3,66 & 0,03 & 3,45 & 0,04 & 5,43 & 0,020 \\
\hline
\end{tabular}


res tiveram mais pontuação na percepção de um clima tarefa e regulação intrínseca com relação aos homens. Estes resultados coincidem com investigações prévias, como as realizadas por Carr \& Weigand ${ }^{50}$, Duda ${ }^{43}$, Duda \& Whitehead ${ }^{51}$, Fortier et al..$^{52}$, Kavussanu \& Roberts ${ }^{53}$, Li et al..$^{54}$ e Ntoumanis \& Biddle ${ }^{55}$, que apontam que as mulheres geralmente apresentam uma orientação à tarefa, percepção de um clima tarefa, motivação intrínseca e motivação identificada, maior do que os homens. Não obstante, as pesquisas indicam que os homens costumam pontuar mais alto em competência esportiva do que as mulheres $4,33,46,56$, ainda que existam alguns estudos que não apreciam tais diferenças ${ }^{57}$.

Desde a Teoria de Metas de Logro ou a Teoria da Autodeterminação é difícil encontrar uma clara explicação às diferenças por sexo encontradas neste estudo. Não obstante, poderíamos sugerir neste caso a influência de outros fatores sociais. Não devemos esquecer que a mulher foi estereotipada como biológica e fisicamente inferior que o homem. Neste sentido, os estereótipos penetrariam dentro das dinâmicas dos centros esportivos e outras instituições, de tal modo que pressionaria os usuários a expressar e demonstrar certas imagens e condutas que satisfazem as expectativas do grupo e da sociedade em gera ${ }^{58,59}$. Dado que o autoconceito físico e, em particular, a competência percebida, se referem às crenças que a pessoa tem sobre o quão hábil é em diferentes domínios de logro, o que pense que tem seria tão, ou mais importante, como o que realmente tenha. E é nesta lógica, onde se pode pensar que as mulheres possivelmente percebam que elas têm menos competência motora que a que realmente têm, isto é, que subestimam sua competência física. De fato, as expectativas criadas pelo centro, grupos de pressão, monitores e outros agentes, poderiam afetar a imagem que homens e mulheres têm de si. Contudo, não devemos esquecer que a construção de uma imagem atlética para uma mulher poderia ser vista em muitos casos como inapropriada ${ }^{54,60}$, de tal forma que as ideologias associadas ao esporte marginariam as mulheres com claras conseqüências negativas para elas. Como demonstrou Weiss \& Horn ${ }^{61}$, as pessoas que subestimam sua competência costumam estar menos motivadas para os desafios e apresentam mais ansiedade em situações de competição a respeito daquelas outras que subestimam sua competência física ou avaliam corretamente a que têm.

Apesar disso, à margem dos possíveis fatores sociais associados às diferenças de gênero, desde a perspectiva de metas de logro e a motivação autodeterminada se podem potenciar uma melhora da percepção motora. Existe suficiente evidência na bibliografia especializada sobre as contribuições que tendem a favorecer ambientes de aprendizagem orientados à tarefa para favorecer mais autonomia ${ }^{10,11}$. Uma maior autodeterminação, como se mostrou neste estudo, estaria associada com a percepção de uma competência motora mais alta. Além disso, os efeitos de um programa de atividade física onde se potencie a autonomia, através de ambientes de aprendizagem, seriam especialmente visíveis em pessoas com baixa auto-estima e autoconceito físico $4,34,62,63,64$

\section{REFERÊNCIAS}

1. Martínez-Tur V, Peiró JM, Ramos J. Efecto modulador de los aspectos sociodemográficos en la predicción de la práctica deportiva y uso de instalaciones deportivas. Anales de psicología. 1995;11(1):77-96.

2. American College of Sports Medicine. The recommended quality and quality exercise for developing a maintaining cardiorrespiratory and muscular fitness in healthy adults. Med Sci Sports Exerc. 1998;30:975-91.

3. American College of Sports Medicine. ACSM's guidelines for exercise testing and prescription. $6^{\text {th }}$ ed. Philadelphia, PA: Lippincott, Williams \& Wilkins; 2000.

4. Marsh HW. The measurement of physical self-concept: a construct validation approach. In: Fox KR, editor. The physical self: from motivation to well-being. Champaign, IL: Human Kinetics; 1997.

5. Raudsepp L, Kristian K, Hannus A. Stability of physical self-perceptions during early adolescence. Pediatr Exerc Sci. 2004;16:138-46.

6. Wigfield A, Eccles JS, Mac lver D, Reuman DA, Midgley C. Transitions during early adolescence: changes in children's domain-specific self-perceptions and general self-esteem across the transition to junior high school. Dev Psychol. 1991;27:552-65.

7. Roberts GC. Understanding the dynamics of motivation in physical activity: the influence of achievement goals on motivational process. In: Roberts GC, editor. Advances in motivation in sport and exercise. Champaign, IL: Human Kinetics; 2001

8. Deci EL, Ryan RM. Intrinsic motivation and self-determination in human behavior. New York: Plenum Press; 1985.

9. Ryan RM, Deci EL. Self-determination theory and the facilitation of intrinsic motivation, social development and well-being. Am Psychol. 2000;55:6878.

10. Ames C. Achievement goals, motivational climate and motivational processes. In: Roberts G, editor. Motivation in Sport and Exercise. Champaign, IL: Human Kinetics; 1992.

11. Ames C. Classrooms, goals, structures and student motivation. J Educ Psychol. 1992;84:261-71.

12. Ames $C$, Ames R. Systems of student and teacher motivation: Towards a qualitative definition. Journal of Educational Psychology, 1984;76:535-56.

13. Dweck, CS. Motivational processes affecting learning. Am Psychol. 1986;41:1040-8.

14. Nicholls JG. Achievement motivation: Conceptions of ability, subjective experience, task choice and performance. Psychol Rev. 1984;21:328-46.

15. Nicholls JG. The competitive ethos and democratic education. Cambridge: Harvard University Press; 1989.

16. Deci EL, Ryan RM. A motivational approach to self: Integration in personality. In: R. Dienstbier (Ed.), Nebraska symposium on motivation. Lincoln, NE: University of Nebraska Press; 1991

17. Deci EL, Ryan RM. The "what" and "why" of goal pursuits: Human needs and the self-determination of behavior. Psychol Inq, 2000;11:227-68.

18. Ntoumanis N. Motivational clusters in a simple of British physical education classes. Psychol Sport and Exerc. 2002;3:177-94.

19. Maehr ML, Nicholls JG. Culture and achievement motivation: A second look. In: Warren N, editor. Studies in cross-cultural psychology. New York: Academic Press; 1980.

20. Elliot AJ. Approach and avoidance motivation and achievement goals. Educ Psychol. 1999;34:169-89. 
21. Elliot AJ, McGregor HA. A $2 \times 2$ achievement goal framework. J Pers Soc Psychol. 2001;80:501-9.

22. Halliburton AL, Weiss MR. Sources of competence information and perceived motivational climate among adolescent female gymnasts varying in skill level. J Sport Exerc Psychol 2002;24:396-419.

23. Krane V, Greenleaf CA, Snow J. Reaching for gold and the price of glory: A motivational case study of an elite gymnast. Sport Psychol. 1997;1 1:5371.

24. Harter S. Effectance motivation reconsidered: toward a developmental model. Hum Dev. 1978;21:34-64.

25. Harter S, Whitesell NR, Kowalski P. Individual differences in the effects of educational transitions on young adolescent's perceptions of competence and motivational orientation. Am Educ Res J. 1992;29:777-807.

26. Valentini N, Rudisill M. Motivational climate, motor-skill development, and perceived competence: two studies of developmentally delayed kindergarten children. J Teach Physical Educ. 2004;23:216-34.

27. Weiss MR, Ferrer-Caja E. Motivational orientations and sport behavior In: Horn TS, editor. Advances in sport psychology. Champaign, IL: Human Kinetics; 2002

28. Weiss MR, Bredemeier BJ, Shewchuk RM. The dynamics of perceived competence, perceived control, and motivational orientation in youth sports In: Weiss MR, Gould D, editors. Sport for children and youths (pp. 89-101). Champaign, IL: Human Kinetics; 1986.

29. Cervelló E, Moreno JA, del Villar F, Reina R. Desarrollo y validación de un instrumento de medida de las estrategias motivacionais empleadas en las clases de educación física. Revista Iberoamericana de Psicología del ejercicio y el Deporte. 2007;2(2):53-72.

30. Guan J, Xiang P, McBride R, Bruene A. Achievement goals, social goals and students' reported persistence and effort in high school physical education. J Teach Physical Educ. 2006;25:58-74.

31. Moreno JA, Cervelló E, Martínez Camacho A. Measuring self-determination motivation in a physical fitness setting: validation of the Behavioral Regulation in Exercise Questionnaire-2 (BREQ-2) in a Spanish sample. J Sports Med Phys Fitness. 2007;47:366-78.

32. Markland D, Tobin V. A modification to Behavioral Regulation in Exercise Questionnaire to include an assessment of amotivation. J Sport Exerc Psychol. 2004;26:191-6.

33. Moreno JA, Cervelló E. Physical self-perception in Spanish adolescents: gender and involvement in physical activity effects. J Hum Mov Stud. 2005:48:291-311

34. Fox KR, Corbin CD. The physical self perception profile: Development and preliminary validation. J Sport Exerc Psychol. 1989;11:408-30.

35. Nunnally JC. Psychometric theory. New York: McGraw-Hill; 1978.

36. Hair JF, Anderson RE, Tatham RL, Black WC. Multivariate data analysis. New Jersey: Prentice-Hall; 1998

37. Nunnally JC, Bernstein IH. Psychometric theory. New York: McGraw-Hill 1994.

38. Cervelló EM, Santos-Rosa FJ. Motivation in sport: an achievement goal perspective in young Spanish recreational athletes. Percept Mot Skills. $2001 ; 92: 527-34$.

39. Goudas M, Biddle S, Fox K. Perceived locus of causality, goal orientations and perceived competence in school in school physical education lessons. Br J Educ Psychol. 1994;64:453-63.

40. Seifriz J, Duda JL, Chi L. The relationship of perceived motivational climate to intrinsic motivation and beliefs about success in basketball. J Sport Exerc Psychol. 1992;14:375-91.

41. White SA, Duda JL. The relationship of gender, level of sport, involvement, and participation motivation to task and ego orientation. Int J Sport Psychol. 1994;25:4-18.

42. Duda JL, Chi L, Newton M, Walling MD, Catley D. Task and ego orientation and intrinsic motivation in sport. Int J Sport Psychol. 1995;26:40-63.

43. Duda JL. Goal perspective research in sport: Pushing the boundaries and clarifying some misunderstandings. En G. Roberts (Ed.), Advances in motivation in sport and exercise (pp. 129-182) Champaign, IL: Human Kinetics; 2001

44. Goñi A, Zulaika LM. Relationships between physical education classes and the enhancement of fifth grade pupils' self-concept. Percept Mot Skills. 2000;91:146-50

45. Hagger M, Biddle S, Wang CK. Physical self-concept in adolescence: generalizability of a multidimensional, hierarchical model across gender and grade. Educ Psychol Meas. 2005;65(2):297-322

46. Maïano C, Ninot G, Bilard J. Age and gender effects on global selfesteem and physical self-perception in adolescents. Eur Phys Educ Rev. 2004; 10:53-69.

47. Georgiadis MM, Biddley SJ, Chatzisarantis NL. The mediating role of selfdetermination in the relationship between goal orientations and physical self-worth in Greek exercisers. Eur J Sport Sci. 2001;1(5):1-9.

48. Reinboth M, Duda JL. The motivational climate, perceived ability, and athletes' psychological and physical well-being. Sport Psychol. 2004;18:23751

49. Duda JL, Kim MS. Perceptions of the motivational climate, psychological characteristics, and attitudes toward eating among young female gymnasts. J Sport Exerc Psychol. 1997;(Suppl):S48.

50. Carr S, Weigand DA. Parental, peer, teacher and sporting hero influence on the goal orientations of children in physical education. Eur Phys Educ Rev. $2001 ; 7: 305-28$.

51. Duda JL, Whitehead J. Measurement of goal perspectives in the physical domain. In: Duda JL, editor. Advances in sport and exercise psychology measurement. Morgantown, WV: Fitness Information Technology; 1998.

52. Fortier MS, Vallerand RJ, Briere NM, Provencher PJ. Competitive and recreational sport structures and gender. A test of their relationship with sport motivation. Int J Sport Psychol. 1995;26:24-39.

53. Kavussanu M, Roberts GC. Motivation in physical activity contexts: the relationship of motivational climate to intrinsic motivation and self-efficacy. J Sport Exerc Psychol. 1996:18;246-80.

54. Li F, Harmer $P_{1}$ Acock $A$. The task and ego orientation in sport questionnaire: Construct equivalence and mean differences across gender. Res $Q$ Exerc Sport. 1996:68(2);228-38.

55. Ntoumanis $\mathrm{N}$, Biddle $\mathrm{S}$. Affect and achievement goals in physical activity: a meta-analysis. Scand J Med Sci Sports. 1999:9;315-32.

56. Gutiérrez M, Moreno JA, Sicilia A. Autoconcepto físico y práctica deportiva de una muestra de estudiantes universitarios. Comunicación presentada en el IV Congrés de les Ciéncies de l'Esport, l'Educació Física I la Recreació. Lleida. INEFC; 1999.

57. Welk GJ, Eklund B. Validation of the children and youth physical selfperception profile for young children. Psychol Sport Exerc. 2005:6;51-65.

58. Davis K. Embodied practices: feminist perspectives on the body. Londres: Sage; 1997.

59. Hall MA. Feminism and sporting bodies: essays on theory and practice. Champaign, IL: Human Kinetics; 1996.

60. Solmon MA, Lee AM, Belcher D, Harrison L, Wells L. Beliefs about gende appropriateness, ability and competence in physical activity. J Teach Phys Educ. 2003:22:261-79.

61. Weiss MR, Horn TS. The relationship between children's accuracy estimates of their physical competence and achievement-related behaviors. Res $Q$ Exerc Sport. 1990;61:250-8.

62. Fox KR. The Physical Self-Perception Profile manual. DeKalb: Northern Illinois University; 1990

63. Hagborg WJ. The Rosenberg self-esteem scale and Harter's self-perception profile for adolescents: a concurrent validity study. Psychol Sch. 1994:30;132-6

64. Marsh HW, Richards GE, Johnson S, Roche L Tremayne P. Physical selfdescription questionnaire: psychometric properties and a multitraitmultimethod analysis of relations to existing instruments. J Sport Exerc Psychol. 1994:16;270-305.

Recebido: 04/04/2008 - Aceito: 17/08/2008 